The Astrophysical Journal, 661:L195-L198, 2007 June 1

(C) 2007. The American Astronomical Society. All rights reserved. Printed in U.S.A.

\title{
DIRECT MEASUREMENT OF THE RADIUS AND DENSITY OF THE TRANSITING EXOPLANET HD 189733b WITH THE CHARA ARRAY
}

\author{
Ellyn K. Baines, ${ }^{1}$ Gerard T. van Belle, ${ }^{2}$ Theo A. Ten Brummelaar, ${ }^{1}$ Harold A. McAlister, ${ }^{1}$ Mark Swain, ${ }^{3}$ \\ Nils H. Turner, ${ }^{1}$ Laszlo Sturmann, ${ }^{1}$ and Judit Sturmann ${ }^{1}$ \\ Received 2007 February 23; accepted 2007 April 20; published 2007 May 9
}

\begin{abstract}
We have measured the angular diameter of the transiting extrasolar planet host star HD 189733 using the CHARA optical/IR interferometric array. Combining our new angular diameter of $0.377 \pm 0.024$ mas with the Hipparcos parallax leads to a linear radius for the host star of $0.779 \pm 0.052 R_{\odot}$ and a radius for the planet of $1.19 \pm 0.08 R_{\text {Jup }}$. Adopting the mass of the planet as derived by its discoverers, we derive a mean density of the planet of $0.91 \pm 0.18 \mathrm{~g} \mathrm{~cm}^{-3}$. This is the first determination of the diameter of an extrasolar planet through purely direct means.
\end{abstract}

Subject headings: infrared: stars — stars: fundamental parameters — stars: individual (HD 189733) — techniques: interferometric

\section{INTRODUCTION}

A handful of extrasolar planets transit their host stars, causing a reduction in stellar flux as the planet blocks part of the star's disk. The planet orbiting HD 189733 is one of the 14 known transiting planets. Using radial velocity and photometric measurements made at the Haute-Provence Observatory, Bouchy et al. (2005) discovered a hot Jupiter-like planet with an orbital period of 2.219 days and estimated the star's radius to be $0.76 \pm$ $0.01 R_{\odot}$. This value, along with a planet-to-star radius ratio of $0.172 \pm 0.003$, led to a planetary radius of $1.26 \pm 0.03 R_{\text {Jup }}$. More recently, Bakos et al. (2006b) refined the orbital parameters using $B V R I$ multiband photometry and found the planet's radius to be $1.154 \pm 0.032 R_{\text {Jup }}$.

We observed HD 189733 using Georgia State University's Center for High Angular Resolution Astronomy (CHARA) Array in order to directly determine the host star's radius and thereby calculate, in a strictly geometric manner, the radius and density for the planet.

Planetary densities were previously estimated from photometric observations of the transiting planets and range from $0.38 \mathrm{~g} \mathrm{~cm}^{-3}$ for HD 209458b (Charbonneau et al. 2000) to $1.17 \mathrm{~g} \mathrm{~cm}^{-3}$ for HD $149026 \mathrm{~b}$ (Sato et al. 2005). These density calculations are highly dependent on estimated stellar diameters based on spectral energy distribution (SED) fits using published photometric values, which are fundamentally indirect in nature, relying upon a priori assumptions regarding the host stars' stellar atmospheres. For the four "bright" $(V<12)$ transit host stars, these angular sizes are in the range of $0.05-0.40$ mas. The longest baselines of the CHARA Array are capable of resolving the largest and brightest of these objects.

\section{INTERFEROMETRIC OBSERVATIONS AND DIAMETER DETERMINATION}

\subsection{Observations and Data Reduction}

Spatially resolved observations of HD 189733 were obtained with the CHARA Array, a six-element interferometer located on

\footnotetext{
${ }^{1}$ Center for High Angular Resolution Astronomy, Georgia State University, P.O. Box 3969, Atlanta, GA 30302-3969; baines@chara.gsu.edu, theo@chara-array .org, hal@chara.gsu.edu, nils@chara-array.org, sturmann@chara-array.org,judit@ chara-array.org.

${ }^{2}$ Michelson Science Center, California Institute of Technology, 770 South Wilson Avenue, MS 100-22, Pasadena, CA 91125; gerard@ipac.caltech.edu.

${ }^{3}$ Jet Propulsion Laboratory, 4800 Oak Grove Drive, Pasadena, CA 91109; mark.swain@jpl.nasa.gov.
}

Mount Wilson, California (McAlister et al. 2005). The Array operates in two wavelength regimes: in visible wavelengths $(470-800 \mathrm{~nm})$ for tracking and tip/tilt correction, and in the nearinfrared $K^{\prime}(2.13 \mu \mathrm{m})$ and $H(1.67 \mu \mathrm{m})$ bands for fringe detection. Because of the small angular diameter for the star, only the $H$ band observations obtained at our longest baseline pair (telescopes E1 and S1) are used in our final diameter analysis.

HD 189733 was observed on several nights during the summer of 2006 along with the calibrator star HD 190993, a B3 $\mathrm{V}$ star offset by $1.7^{\circ}$, selected on the basis of its small estimated angular diameter and its apparent lack of any close companion. The latter criterion was verified by a thorough literature search, while a SED fit to HD 190993 led to an estimated angular diameter of $0.167 \pm 0.035$ mas with no residuals suggestive of a companion (see Fig. 1). This results in a predicted visibility $(V)$ for the calibrator of $V_{\text {cal }}=0.961_{-0.008}^{+0.019}$ at our longest baseline of $330 \mathrm{~m}$, resulting in a contribution of $\sigma_{V} \simeq 0.01-0.02$ to the calibrated visibility errors seen in Table 1 . The small angular size and high visibility of the calibrator mean that HD 190993 is essentially unresolved using the CHARA Array, and the uncertainty in visibility due to calibrator diameter error is small compared to the measurement error. Therefore, uncertainties in the calibrator diameter will not affect the HD 189733 diameter measurement significantly (van Belle \& van Belle 2005). Even HD 190993's considerable $v \sin i$ does not contribute an error to our diameter fits due to its small angular size.

We note that the M dwarf companion to HD 189733 reported by Bakos et al. (2006a) on the basis of common space motion at an angular separation of $11.2^{\prime \prime}$ is well outside the interferometric field of view, and its presence has no effect on our results. Although the effect on visibility would be small in the first lobe of the $V(B)$ curve, we have confirmed that our observed epochs do not occur within the predicted times of planetary transit or eclipse using the period and reference time of central transit of Bakos et al. (2006b).

All our observations were obtained with the single-baseline, pupil-plane "CHARA Classic" beam combiner, and we employed the standard practice of observing the target and calibrator sequentially to provide a series of time-bracketed observations from which the instrumental visibilities could be reduced to calibrated values for the target star. The observing practice and reduction process employed here are identical to 
TABLE 1

HD 190993--B3V Net SED Model

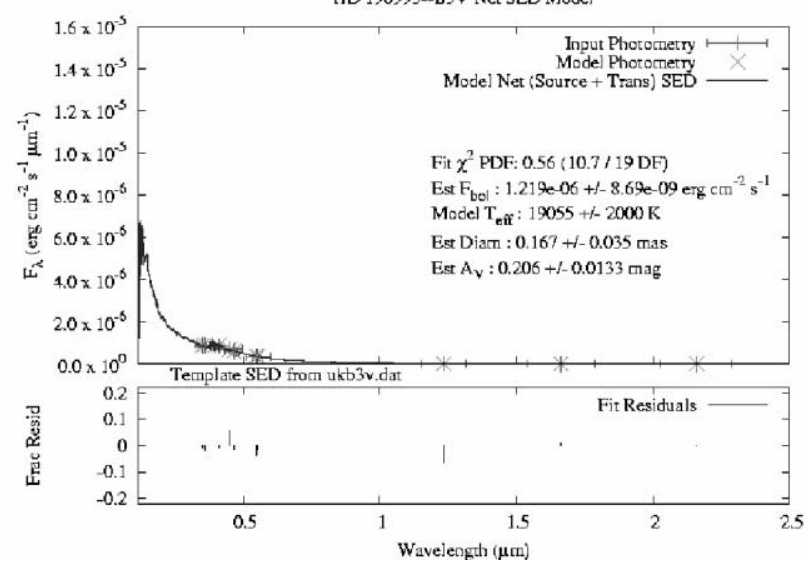

FIG. 1.-SED fit of our calibrator star, HD 190993. In the top panel, the vertical bars represent the errors for the data points that they overlay, and the horizontal bars represent the bandpass of the data points. In the bottom panel, the fractional residuals (difference between data point and fit point, normalized by that data point) are shown for each data point.

those described by ten Brummelaar et al. (2005). The results of this process are summarized in Table 1.

Single-baseline Michelson stellar interferometers measure complex visibilities, usually recorded as amplitudes and phases, which are related to the intensity distribution of the target through a Fourier transform; phase information is typically corrupted by the atmosphere, leaving the amplitude, referred to simply as the visibility (Colavita et al. 1999).

\subsection{Diameter Fit}

Diameter fits to the visibilities and baselines from Table 1 were performed using the uniform-disk (UD) approximation given by

$$
V=\frac{2 J_{1}(x)}{x}
$$

where $J_{1}$ is the first-order Bessel function and

$$
x=\pi B \theta_{\mathrm{UD}} \lambda^{-1},
$$

where $B$ is the projected baseline at the star's position, $\theta_{\mathrm{UD}}$ is the apparent UD angular diameter of the star, and $\lambda$ is the wavelength of the observation. The limb-darkened (LD) relationship incorporating the linear limb-darkening coefficient $\mu_{\lambda}$ (Hanbury Brown et al. 1974) is given by

$$
V=\left(\frac{1-\mu_{\lambda}}{2}+\frac{\mu_{\lambda}}{3}\right)^{-1}\left[\left(1-\mu_{\lambda}\right) \frac{J_{1}(x)}{x}+\mu_{\lambda}\left(\frac{\pi}{2}\right)^{1 / 2} \frac{J_{3 / 2}(x)}{x^{3 / 2}}\right] .
$$

These fits resulted in $\Theta_{\mathrm{UD}}=0.366 \pm 0.024$ mas and $\Theta_{\mathrm{LD}}=$ $0.377 \pm 0.024$ mas, the latter incorporating $\mu_{\lambda}=0.36$ taken from Claret et al. (1995) after adopting $\log g=4.5$ and $T_{\text {eff }}=5000 \mathrm{~K}$ for HD 189733 (see Fig. 2). The reduced $\chi^{2}$ minimization in both cases yielded a value of 1.593 , and the errors quoted are for an increase of the $\chi^{2}$ value of 1.0, that is, the $68 \%$ confidence interval. Dividing this $\chi^{2}$ by the number of degrees of freedom, which in our case is 8 , yields 0.199 , which is much less than 1.0 , showing that the fit is quite good
INTERFERometric MEASUREMENTS OF HD 189733

\begin{tabular}{cccc}
\hline \hline $\begin{array}{c}\text { MJD } \\
(53,886.0+)\end{array}$ & $\begin{array}{c}\text { Baseline } \\
(\mathrm{m})\end{array}$ & Visibility & $\sigma_{V}$ \\
\hline $0.905 \ldots \ldots \ldots \ldots \ldots$ & 330.5 & 0.851 & 0.071 \\
$1.936 \ldots \ldots \ldots \ldots \ldots$ & 327.9 & 0.843 & 0.056 \\
$1.958 \ldots \ldots \ldots \ldots \ldots$ & 324.9 & 0.857 & 0.054 \\
$8.865 \ldots \ldots \ldots \ldots \ldots$ & 330.5 & 0.869 & 0.034 \\
$76.742 \ldots \ldots \ldots \ldots \ldots$ & 326.5 & 0.909 & 0.069 \\
$76.761 \ldots \ldots \ldots \ldots \ldots$ & 323.8 & 0.863 & 0.049 \\
$76.778 \ldots \ldots \ldots \ldots \ldots$ & 321.3 & 0.877 & 0.045 \\
$76.793 \ldots \ldots \ldots \ldots \ldots$ & 319.0 & 0.839 & 0.045 \\
$76.824 \ldots \ldots \ldots \ldots \ldots$ & 315.5 & 0.829 & 0.061 \\
\hline
\end{tabular}

and that our error estimates for the visibility points are conservative. If we rescale these error bars to force $\chi^{2}$ to be equal to the number of degrees of freedom, which assumes that there are no systematics in the measurements, they are approximately half the size that they are shown in Figure 2 and would also reduce our final error estimates by a factor of 2 . However, we will remain conservative and continue to use the error estimate based on the raw $\chi^{2}$ value.

\subsection{Estimate of the Angular Size of HD 189733}

An a priori estimate of the angular size of HD 189733 is a parameter of considerable interest, because the size of HD $189733 \mathrm{~b}$ is determined only relative to the size of its parent star from the photometric transit timing data. Bakos et al. (2006b) consider no less than four separate methods in their investigation of the system: the $V-K$ color angular radius prediction (Kervella et al. 2004), the temperature radius, isochrone radii from Girardi et al. (2002) and Baraffe et al. (1998), and the Johnson $V-2$ MASS $T_{\text {eff }}$ calibration of Masana et al. (2006).

None of these approaches appear to have much merit, since the only primary data we have been able to find in the literature were Tycho $B_{\mathrm{T}}, V_{\mathrm{T}}$ (Bessell 2000), Strömgren ubvy (Olsen 1993), and 2MASS JHK photometry (Cutri et al. 2003). Neither spectroscopy nor measures of $\log g$ nor direct measures of Johnson photometry appear to be available in the literature. The values of $V$ used in Kervella et al. (2004) and Masana et al. (2006) appear to have been extrapolated from $V_{\mathrm{T}}$. Furthermore, sustained long-term observations of HD 189733 by the MOST asteroseismology satellite have found the star to be pho-

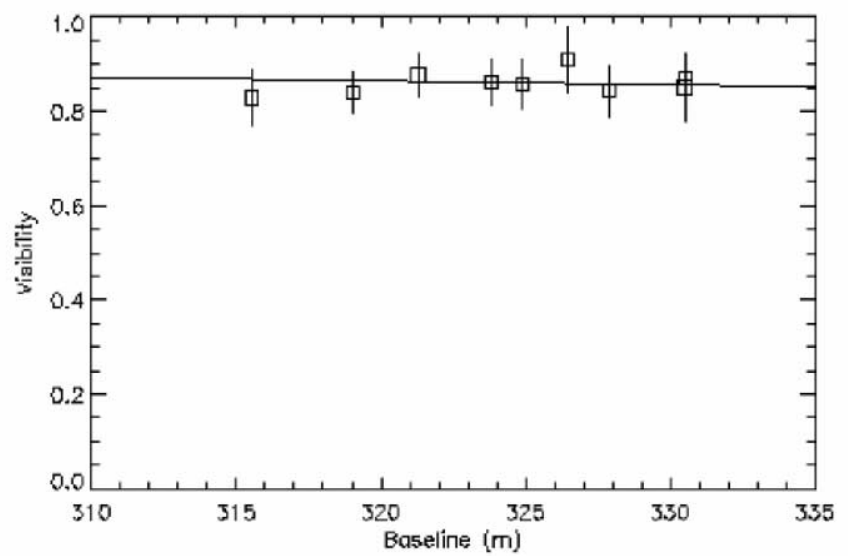

FIG. 2. - The $\chi^{2}$ fit to HD 189733 's visibilities. The solid line represents a theoretical visibility curve for a star with a limb-darkened diameter of 0.377 mas, the squares are the measured visibilities, and the vertical lines are the measured errors. 
tometrically variable (J. Rowe 2007, private communication), casting significant doubt on any radius derived from a photometric relationship. Based on this information, we consider the size errors for HD 189733 quoted in Bouchy et al. (2005) and Bakos et al. (2006b), derived from the methods cited above, to be underestimates.

To explore what is a more appropriate error for an inferred angular size, we executed a SED fit of the available spectrophotometry for HD 189733 cited above. Given the known variability of HD 189733, the quoted millimagnitude error estimates of the Tycho and Strömgren photometric data points were increased by a factor of 10 . These photometric data points were fit to the solar-abundance $\mathrm{K} 0 \mathrm{~V}$ and $\mathrm{K} 2 \mathrm{~V}$ templates available from Pickles (1998), with the resulting fit values for reddening, bolometric flux, and angular diameter seen in Table 2, along with the appropriate $\chi^{2}$ per degree of freedom $\left(\chi^{2}\right.$ PDF) values. Unfortunately, a K1 V template is not available in that library, although an estimate of one from interpolating between the two bracketing spectral types was synthesized by us for testing this spectral type. These fits are seen in Figure 3.

The appropriate model spectrum from Munari et al. (2005) for a $5000 \mathrm{~K}$ star was fit with a $\chi^{2}$ PDF of 2.80 , but this model (and the others available in the literature) unfortunately only covered the visible portion of the spectrum. The $115-2500 \mathrm{~nm}$ range of Pickles (1998) was necessary to fully characterize the available photometry, and thus we constrained our analysis to this particular set of stellar templates.

Our finding is that, even with this highly detailed analysis of the stellar SED, the most appropriate modeling of that SED reveals a predicted angular size of only $\theta=0.363 \pm 0.011$ mas-a $3 \%$ error bar-which corresponds to a stellar linear radius of $R=$ $0.752 \pm 0.026 R_{\odot}$.

\section{DISCUSSION}

Our new direct determination for the angular diameter of HD 189733 of $\Theta_{\mathrm{LD}}=0.377 \pm 0.024$ mas can be combined with the Hipparcos parallax for the star of $\pi=51.9 \pm 0.9$ mas (Perryman et al. 1997) to give a physical radius for the star of $R_{\text {star }}=0.779 \pm 0.052 R_{\odot}$, which is about $3 \%$ larger than that adopted by Bakos et al. (2006b).

By the nature of the light-curve analysis, the relative increase in the radius of the host star will directly translate into the same relative increase in the radius of the planet HD $189733 \mathrm{~b}$. Thus, revising the radius of Bakos et al. (2006b) of $1.154 R_{\mathrm{Jup}}$, our new estimate for this value is $R_{\text {planet }}=1.19 \pm 0.08 R_{\text {Jup }}$. Furthermore, adopting the value of Bouchy et al. (2005) for the mass of the planet of $1.15 M_{\text {Jup }}$, we derive a new estimate for the density of HD 189733b, $\rho=0.91 \pm 0.18 \mathrm{~g} \mathrm{~cm}^{-3}$. These values are in good agreement with Winn et al. (2007), who used transit photometry to constrain the stellar and planetary radii. The values of $M_{\text {planet }}, R_{\text {planet }}$, and $\rho_{\text {planet }}$ are all consistent with the modest collection of these parameters presently available for transiting exoplanet systems, and they support the conclusion that HD 189733 is not among the few hot Jupiters that present extraordinarily large radii for their masses.

\section{INTERFEROMETRIC NONDETECTION OF BINARITY OF HD 189733}

Given the higher resolution of interferometric arrays, a possible close-separation tertiary companion may affect our measures of HD 189733's visibility and thereby complicate our interpretation. As such, it was prudent for us to also observe
HD 189733 with the Palomar Testbed Interferometer (PTI; Colavita et al. 1999), an instrument with intermediate baselines on a variety of sky projections, suitable for exploration of possible unseen nearby luminous (stellar) companions. The PTI has been demonstrated to be sensitive to nearby companions with $\Delta K<4.0$ (Boden et al. 1998), which for a K2-3 V primary star rules out any M dwarf companions (Bessell \& Brett 1988).

PTI observed HD 189733 in the $K$ band on the nights of 2006 June 10-12, 2006 June 24, and 2006 July 8-10. Four of those nights used PTI's 85 m northwest baseline configuration, two used the $110 \mathrm{~m}$ north-south baseline, and one night was
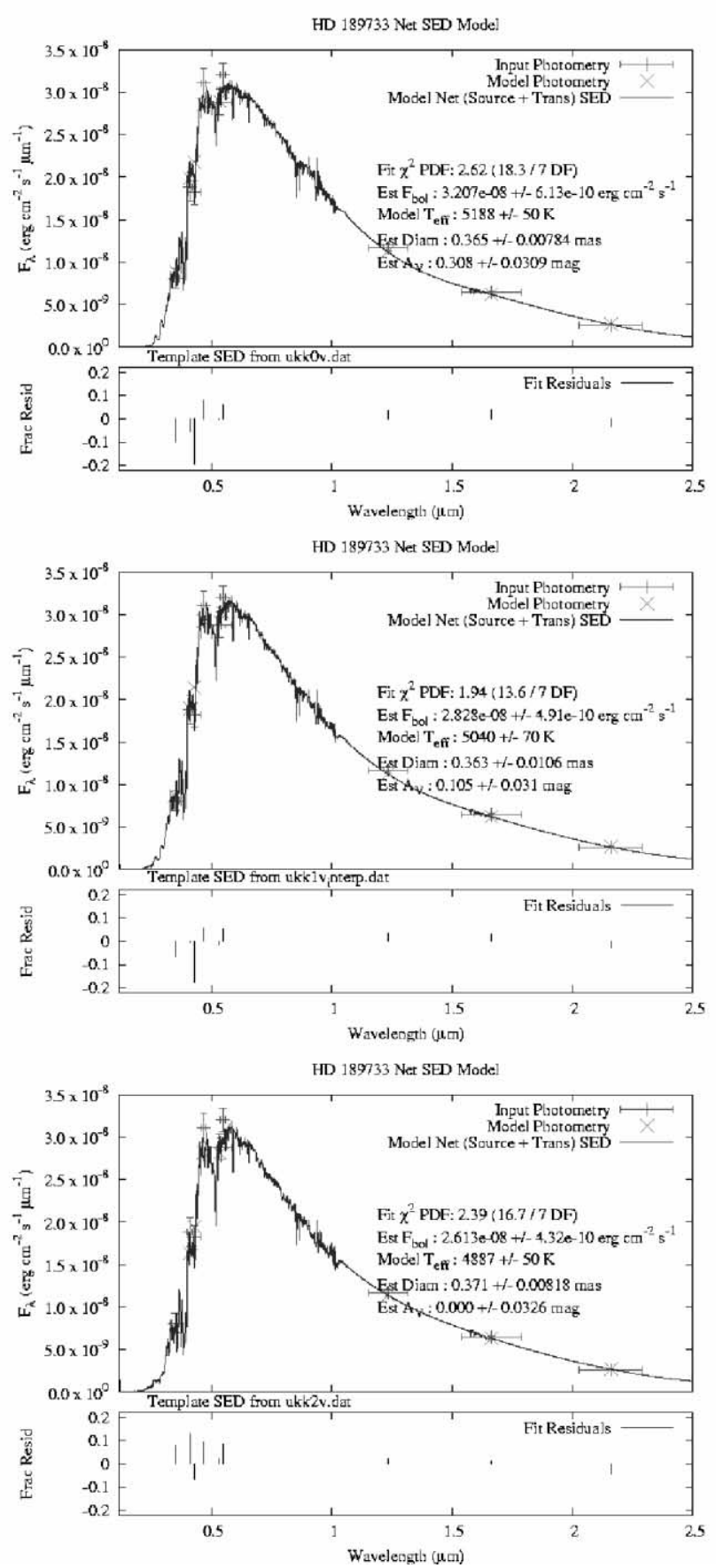

FIG. 3.-SED fits of HD 189733. The top panel shows the K0 V photometric fit, the middle panel shows the K1 V fit, and the bottom panel shows the K2 $\mathrm{V}$ fit. All three fits allowed the reddening factor to vary. 
TABLE 2

SED Fits for HD 189733 Photometry to Emperical Spectral Templates

\begin{tabular}{|c|c|c|c|c|c|}
\hline \multicolumn{2}{|c|}{ Model Parameters ${ }^{\mathrm{a}}$} & \multicolumn{4}{|c|}{ FitTed PARAMETERs $^{\mathrm{b}}$} \\
\hline Spectral Type & $\begin{array}{l}T_{\text {eff }} \\
(\mathrm{K})\end{array}$ & $\chi^{2} \mathrm{PDF}$ & $\begin{array}{l}\text { Reddening } A_{V} \\
\text { (mag) }\end{array}$ & $\begin{array}{c}F_{\text {bol }} \\
\left(10^{-8} \mathrm{ergs} \mathrm{cm}^{-2} \mathrm{~s}^{-1} \mu \mathrm{m}^{-1}\right)\end{array}$ & $\begin{array}{c}\theta \\
(\mathrm{mas})\end{array}$ \\
\hline $\mathrm{K} 0 \mathrm{~V}$ & $5188 \pm 50$ & 2.62 & $0.308 \pm 0.031$ & $3.207 \pm 0.061$ & $0.365 \pm 0.008$ \\
\hline $\mathrm{K} 1 \mathrm{~V}^{\mathrm{c}}$ & $5040 \pm 70$ & 1.94 & $0.105 \pm 0.031$ & $2.828 \pm 0.049$ & $0.363 \pm 0.011$ \\
\hline $\mathrm{K} 2 \mathrm{~V} \ldots \ldots \ldots$ & $4887 \pm 50$ & 2.39 & $0.00 \pm 0.030$ & $2.613 \pm 0.043$ & $0.371 \pm 0.008$ \\
\hline
\end{tabular}

${ }^{a}$ Models from Pickles (1998); $T_{\text {eff }}$ is the effective temperature.

${ }^{\mathrm{b}} F_{\text {bol }}$ is the bolometric flux, and $\theta$ is the angular diameter.

${ }^{\mathrm{c}} \mathrm{K} 1 \mathrm{~V}$ model interpolated from the $\mathrm{K} 0 \mathrm{~V}$ and $\mathrm{K} 2 \mathrm{~V}$ models.

a 85 m southwest baseline. For all of these nights, HD 189733's normalized $V$ data points were indistinguishable from unit visibility, which corresponds to a completely unresolved point source, as would be expected for a single $\sim 0.37$ mas star being observed by PTI at $2.2 \mu \mathrm{m}$.

\section{CONCLUSION}

Our results for the radii of the host star and planet in the HD 189733 exoplanet system are formally in good agreement with existing measurements of these parameters as well as with the estimate for the density of the planet, and they have the additional and significant merit that they represent direct measurements of stellar and planetary diameters that do not rely on inferences about stellar atmospheres. While the diameter measurements are currently at a $6 \%$ level of accuracy, we expect to improve this considerably as we implement fringe detection at shorter wavelengths at the CHARA Array. In the meantime, these results demonstrate a new role that long-baseline optical/infrared interferometry can play in the field of exoplanet astronomy.

We would like to thank Andy Boden for sharing his SED fit tools with us, which we used to produce the fits seen in
Figures 1 and 3, and we appreciate the care that CHARA Array operator P. J. Goldfinger used in obtaining many of these observations. The CHARA Array is funded by the National Science Foundation through NSF grants AST-0307562 and AST06006958 and by Georgia State University through the College of Arts and Sciences and the Office of the Vice President for Research. Observations with PTI are made possible through the efforts of the PTI Collaboration, which we acknowledge. Funding for PTI was provided to the Jet Propulsion Laboratory under its TOPS (Towards Other Planetary Systems), ASEPS (Astronomical Studies of Extrasolar Planetary Systems), and Origins programs and from the JPL Director's Discretionary Fund. Part of the work described in this Letter was performed at the Jet Propulsion Laboratory under contract with the National Aeronautics and Space Administration. This research has made use of the SIMBAD literature database, operated at CDS, Strasbourg, France, and of NASA's Astrophysics Data System. This publication makes use of data products from the Two Micron All Sky Survey (2MASS), which is a joint project of the University of Massachusetts and the Infrared Processing and Analysis Center/California Institute of Technology, funded by the National Aeronautics and Space Administration and the National Science Foundation.

\section{REFERENCES}

Bakos, G. Á., et al. 2006a, ApJ, 641, L57 2006b, ApJ, 650, 1160

Baraffe, I., et al. 1998, A\&A, 337, 403

Bessell, M. S. 2000, PASP, 112, 961

Bessell, M. S., \& Brett, J. M. 1988, PASP, 100, 1134

Boden, A. F., et al. 1998, ApJ, 504, L39

Bouchy, F., et al. 2005, A\&A, 444, L15

Charbonneau, D., et al. 2000, ApJ, 529, L45

Claret, A., Diaz-Cordoves, J., \& Gimenez, A. 1995, A\&AS, 114, 247

Colavita, M. M., et al. 1999, ApJ, 510, 505

Cutri, R. M., et al. 2003, The IRSA 2MASS All-Sky Point Source Catalog

(Pasadena: NASA/IPAC), http://irsa.ipac.caltech.edu/applications/Gator/

Girardi, L., et al. 2002, A\&A, 391, 195
Hanbury Brown, R., et al. 1974, MNRAS, 167, 475

Kervella, P., et al. 2004, A\&A, 426, 297

Masana, E., Jordi, C., \& Ribas, I. 2006, A\&A, 450, 735

McAlister, H. A., et al. 2005, ApJ, 628, 439

Munari, U., et al. 2005, A\&A, 442, 1127

Olsen, E. H. 1993, A\&AS, 102, 89

Perryman, M. A. C., et al. 1997, A\&A, 323, L49

Pickles, A. J. 1998, PASP, 110, 863

Sato, B., et al. 2005, ApJ, 633, 465

ten Brummelaar, T. A., et al. 2005, ApJ, 628, 453

van Belle, G. T., \& van Belle, G. 2005, PASP, 117, 1263

Winn, J. N., et al. 2007, AJ, 133, 1828 